\title{
Many New Exact Solutions for Generalized Davey-Stewartson Equation with Arbitrary Power Nonlinearities Using Novel $\left(\frac{G^{\prime}}{G}\right)$-Expansion Method
}

\author{
Beibei Zhang ${ }^{1}$, Mei Xiong $^{1 *}$ and Longwei Chen ${ }^{1}$ \\ ${ }^{1}$ College of Statistics and Mathematics, Yunnan University of Finance and Economics \\ Email: clwxmff@163.com
}

\begin{abstract}
The novel $\left(\frac{G^{\prime}}{G}\right)$-expansion method is applied to solve generalized Davey-Stewartson equations with arbitrary power nonlinearities and obtain some exact traveling wave solutions. Via this method, we obtain kink wave solutions, anti-kink wave solutions, exact solitary wave solutions, periodic wave solutions. Also, it is shown that this method is influential for solving nonlinear partial differential equations (PDEs) in mathematical physics and engineering.
\end{abstract}

Keywords: Novel $\left(\frac{G^{\prime}}{G}\right)$-expansion method, Davey-Stewartson equation, exact traveling wave, solitary wave.

\section{Introduction}

In recent years, the study of the exact traveling wave solutions for nonlinear PDEs plays an important role in the area of solid state physics, optical fibers, plasma elastic media. Finding some exact traveling wave solutions has made great progression in mathematicians and physicists. Lots of methods have been presented, such as the inverse scattering method, Darboux transformation, the Hirota bilinear method, the homogeneous balance method and the tanh method [1]-[32]. Moreover, Kudryashov proposed $\left(G^{\prime} / G\right)$-expansion method to obtain some new exact traveling wave solutions of PDEs [33], which is effective.

In this paper, we will use the novel $\left(\frac{G^{\prime}}{G}\right)$-expansion method[34] to receive some new exact solutions for the following generalized Davey-Stewartson equations with arbitrary power nonlinearities[35]:

$$
\left\{\begin{array}{l}
i u_{t}+u_{x x}+u_{y y}+\gamma|u|^{p} u+\alpha u v+\delta|u|^{2 p} u=0 \\
v_{x x}+v_{y y}-\beta\left(|u|^{p}\right)_{x x}=0
\end{array}\right.
$$

where $\alpha, \beta, \gamma, \delta$ are real parameters, $p$ is a positive integer, $v$ is a real function, $u$ is a complex function. Generalized Davey-Stewartson equation with arbitrary power nonlinearities is effective, which describes the short-wave and long-wave motion in water with limited depth. Many researchers have applied a lot of methods for solving Eq.(1.1). For example, Ming Song[35] and Cao Jun[36] used the bifurcation method of dynamical systems to obtain the traveling wave solutions for Eq.(1.1). M Mirzazadeh applied the trial equation method and the ansatz approach to establish solitary waves soliton, dark soliton and singular solitary waves soliton solutions of the Davey-Stewartson equation[37]. Ming Song studied the Davey-Stewartson equation with power law nonlinearity and carried out several different solutions for the bifurcation analysis[38]. Reza Farshbaf Zinati used He's semi-inverse variational principle method (SIVPM), the improved $\tan (\phi / 2)$-expansion method (ITEM) and generalized $\left(G^{\prime} / G\right)$-expansion method (GGM) for seeking more exact solutions of the DS equation[39]. The generalized Kudryashov method is introduced to obtain new soliton solutions of the Davey-Stewartson equation with power law nonlinearity by Seyma Tuluce Demiray[40]. Mehdi Fazli Aghdaei applied the generalized $\tan (\phi / 2)$ method and He's semi-inverse variational method (HSIVM) to seek the exact solitary wave solutions of the Davey-Stewartson equation with power law nonlinearity[41]. 
This paper is organized as follows: In sections 2, we mainly describe the novel $\left(\frac{G^{\prime}}{G}\right)$-expansion method. In sections 3, we apply this method to solve Eq.(1.1). Some conclusions are given in sections 4.

\section{Description of the Novel $\left(\frac{G^{\prime}}{G}\right)$-Expansion Method}

Consider the following nonlinear PDEs:

$$
P\left(t, x_{i}, u_{t}, u_{x_{i}}, u_{x_{i} x_{i}}, u_{x_{i} x_{j}}, u_{t t}, \ldots\right)
$$

where $i, j,=1,2, \ldots, n . \mathrm{P}$ is a polynomial in $u(x, t), u(x, t)$ is an unknown function. The main steps of the novel $\left(\frac{G^{\prime}}{G}\right)$-expansion method are as follows:

Step 1. Make a transformation:

$$
u\left(t, x_{1}, u_{2}, \cdots, u_{n}\right)=\phi(\xi), \xi=\sum_{i=1}^{n} k_{i} x_{i}-c t
$$

Eq.(2.1) can be reduced to the following nonlinear ordinary differential equations(ODEs):

$$
Q\left(u, u^{\prime}, u^{\prime \prime}, u^{\prime \prime \prime}, \ldots\right)=0
$$

where $\mathrm{Q}$ is a function of $u(\xi)$ and its derivatives.

Step 2. Suppose the solution of Eq.(2.3) can be denoted by a polynomial in $\psi(\xi)$ :

$$
u(\xi)=\sum_{j=-n}^{n} \alpha_{j}(\psi(\xi))^{j}
$$

where

$$
\psi(\xi)=d+\frac{G^{\prime}(\xi)}{G(\xi)}
$$

The constants $\alpha_{-n}$ and $\alpha_{n}$ could not be zero simultaneously. $\alpha_{j}(j=0, \pm 1, \pm 2, \ldots, \pm N)$ and $d$ are constants.

Step 3. Consider the second order nonlinear ODE:

$$
G G^{\prime \prime}=\lambda G G^{\prime}+\mu G^{2}+v\left(G^{\prime}\right)^{2}
$$

where prime denotes the derivative with respect $\xi . \lambda, \mu$ and $c$ are real parameters.

The Cole-Hopf transformation $\Phi(\xi)=\frac{G^{\prime}(\xi)}{G(\xi)}$ reduces Eq.(2.6)to the following equation:

$$
\Phi^{\prime}(\xi)=\mu+\lambda \Phi(\xi)+(c-1) \Phi^{2}(\xi)
$$

Thus, Eq.(2.7) has individual twenty five solutions (see[42]).

Step 4. The value of the positive integer $n$ can be determined by balancing the higher order linear terms with nonlinear terms of the higher order occurring in Eq.(2.3).

Step 5. Substitute Eq.(2.4) along with Eq.(2.5)and Eq.(2.6)into Eq.(2.3). Then, obtain polynomials in $\left(d+\frac{G^{\prime}(\xi)}{G(\xi)}\right)$ and $\left(d+\frac{G^{\prime}(\xi)}{G(\xi)}\right)^{-1},(j=0,1,2, \ldots, N)$. Furthermore, collect the coefficients of the resulted polynomials to be zero and receive a system of algebraic equations by Maple. Finally, we deserve the exact solutions of Eq.(2.3). 


\section{Applications of Novel (G'/G)-Expansion Method}

In this section, we use the novel (G'/G)-expansion method to solve Eq.(1.1). First, we suppose Eq.(1.1) as follows:

$$
u(x, y, t)=f(\xi) e^{i \eta}, v(x, y, t)=f_{0}(\xi),
$$

where $\xi=x+y-2(k+\lambda) t, \eta=k x+\lambda y-w t . \lambda, k, w$ are real parameters, $f(\xi)$ is real function.

Making Eq.(3.1) into Eq.(1.1), then letting real and imaginary part be zero respectively:

$$
\left\{\begin{array}{l}
\left(w-k^{2}-\lambda^{2}\right) f+2 f^{\prime \prime}+\gamma f^{p+1}+\alpha f f_{0}+\delta f^{2 p+1}=0, \\
f_{0}=\frac{\beta}{2} f^{p} .
\end{array}\right.
$$

Substituting the second equation of (3.2) into the first equation of (3.2), we obtain

$$
f^{\prime \prime}=-\frac{1}{2} \delta f^{2 p+1}-\frac{1}{2}\left(\gamma+\frac{\alpha \beta}{2}\right) f^{p+1}+\frac{1}{2}\left(\lambda^{2}+k^{2}-w\right) f .
$$

For simplification, we draw

$$
f^{\prime \prime}+k_{5} f^{5}+k_{3} f^{3}+k_{1} f=0,
$$

where $k_{5}=\frac{1}{2} \delta, k_{3}=\frac{1}{2}\left(\gamma+\frac{\alpha \beta}{2}\right), k_{1}=-\frac{1}{2}\left(\lambda^{2}+k^{2}-w\right), p=1$. From Eq.(3.4), we obtain

$$
p \Phi^{\prime \prime}+q \Phi+2 \Phi^{3}=0
$$

where $p=\frac{2}{k_{3}}, q=\frac{2 k_{1}}{k_{3}}$. Substituting (3.1) into (3.5) and balancing the higher order derivative $u^{\prime \prime}$ with the nonlinear term of the highest order $u^{3}$, we receive $n=1$. Thus, the solution of Eq.(3.5) have

$$
u(\xi)=\alpha_{-1}(\Psi(\xi))^{-1}+\alpha_{0}+\alpha_{1}(\Psi(\xi)) .
$$

Inserting Eq.(3.6) into Eq.(3.5), the left hand side becomes polynomials of $\left(d+\frac{G^{\prime}(\xi)}{G(\xi)}\right)$ and $(d+$ $\left.\frac{G^{\prime}(\xi)}{G(\xi)}\right)^{-1},(j=0,1,2, \ldots, N)$. Setting the coefficients of these polynomials to be zero, we deserve a set of algebraic equations for $\alpha_{1}, \alpha_{0}, \alpha_{-1}, d$ and $c$ as follows:

$$
\begin{aligned}
& \left(\frac{G^{\prime}}{G}\right)^{6}: 2 p \alpha_{1}(v-1)^{2}+2 \alpha_{1}^{3}=0 \\
& \left(\frac{G^{\prime}}{G}\right)^{5}: p\left[3 \alpha_{1} \lambda(v-1)+6 \alpha_{1} d(v-1)^{2}\right]+2\left(6 d \alpha_{1}^{3}+3 \alpha_{0} \alpha_{1}^{2}\right)=0 \\
& \left(\frac{G^{\prime}}{G}\right)^{4}: p\left[\alpha_{1}\left(2 \mu v-2 \mu+\lambda^{2}\right)+6 \alpha_{1} d \lambda(v-1)+6 \alpha_{1} d^{2}(v-1)^{2}\right]+2\left(15 d^{2} \alpha_{1}^{3}+15 d \alpha_{0} \alpha_{1}^{2}\right. \\
& \left.\quad+3 \alpha_{1}^{2} \alpha_{-1}+3 \alpha_{1} \alpha_{0}^{2}\right)+q \alpha_{1}=0 \\
& \left(\frac{G^{\prime}}{G}\right)^{3}: p\left[\alpha_{1} \lambda \mu+3 \alpha_{1} d\left(2 \mu v-2 \mu+\lambda^{2}\right)+\left(9 \lambda \alpha_{1} d^{2}+\alpha_{-1} \lambda\right)(v-1)+2(v-1)^{2}\left(\alpha_{1} d^{3}\right.\right. \\
& \left.\left.\quad-\alpha_{-1} d\right)\right]+2\left(20 d^{3} \alpha_{1}^{3}+30 d^{3} \alpha_{0} \alpha_{1}^{2}+12 d \alpha_{1}^{2} \alpha_{-1}+12 d \alpha_{0}^{2} \alpha_{1}+6 \alpha_{0} \alpha_{1} \alpha_{-1}\right. \\
& \left.\quad+\alpha_{0}^{3}\right)+q=0, \\
& \left(\frac{G^{\prime}}{G}\right)^{2}: p\left[3 \alpha_{1} d \lambda \mu+\left(3 \alpha_{1} d^{2}+\alpha_{-1}\right)\left(2 \mu v-2 \mu+\lambda^{2}\right)+3 \lambda(v-1)\left(\alpha_{1} d^{3}-\alpha_{-1} d\right)\right] \\
& \quad+2\left[15 d^{4} \alpha_{1}^{3}+30 d^{3} \alpha_{0} \alpha_{1}^{2}+18 d^{2} \alpha_{-1} \alpha_{1}^{2}+18 d^{2} \alpha_{1} \alpha_{0}^{2}+18 d \alpha_{-1} \alpha_{0} \alpha_{1}\right. \\
& \left.\quad+3 d \alpha_{0}^{2}+3 \alpha_{1} \alpha_{-1}^{2}+3 \alpha_{-1} \alpha_{0}^{2}\right]+q\left(\alpha_{0}+3 \alpha_{0} d+6 \alpha_{1} d^{2}\right)=0,
\end{aligned}
$$




$$
\begin{aligned}
\left(\frac{G^{\prime}}{G}\right)^{1}: & p\left[3 \alpha_{1} \lambda \mu d^{2}+3 \alpha_{-1} \lambda \mu+\left(2 \mu v-2 \mu+\lambda^{2}\right)\left(\alpha_{1} d^{3}-\alpha_{-1} d\right)\right]+2\left[6 d^{5} \alpha_{1}^{3}+15 \alpha_{0} \alpha_{1}^{2} d^{4}\right. \\
& +12 \alpha_{-1} \alpha_{1}^{2} d^{3}+12 \alpha_{1} \alpha_{0}^{2} d^{3}+18 \alpha_{1} \alpha_{0} \alpha_{-1} d^{2}+3 \alpha_{0}^{3} d^{2}+6 \alpha_{1} \alpha_{-1}^{2} d+6 \alpha_{-1} \alpha_{0}^{2} d \\
& \left.+3 \alpha_{0} \alpha_{-1}^{2}\right]+q\left[2 \alpha_{1} d+3 \alpha_{0} d^{2}+4 \alpha_{1} d^{3}\right]=0 \\
\left(\frac{G^{\prime}}{G}\right)^{0}: & p\left[\lambda \mu\left(\alpha_{1} d^{3}-\alpha_{-1} d\right)+2 \alpha_{-1} \mu^{2}\right]+2\left[\alpha_{1}^{3} d^{6}+3 \alpha_{0} \alpha_{1}^{2} d^{5}+3 \alpha_{-1} \alpha_{1}^{2} d^{4}+3 \alpha_{1} \alpha_{0}^{2} d^{4}\right. \\
& \left.+6 \alpha_{0} \alpha_{1} \alpha_{-1} d^{3}+\alpha_{0}^{3} d^{3}+3 \alpha_{1} \alpha_{-1}^{2} d^{2}+3 \alpha_{-1} \alpha_{0}^{2} d^{2}+3 \alpha_{0} \alpha_{-1}^{2} d+\alpha_{-1}^{3}\right]+q\left(\alpha_{1} d^{2}\right. \\
& \left.+\alpha_{0} d^{3}+\alpha_{1} d^{4}\right)=0 .
\end{aligned}
$$

Solving the algebraic equations by Maple ,we obtain

\section{Case 1:}

$$
\begin{gathered}
\alpha_{1}= \pm 2(v-1) \sqrt{\frac{q}{8 \mu(v-1)-2 \lambda^{2}}} \\
\alpha_{0}= \pm(\lambda-2 d(v-1)) \sqrt{\frac{q}{8 \mu(v-1)-2 \lambda^{2}}} \\
\alpha_{-1}=0 \\
p=\frac{-2 q}{4 \mu(v-1)-\lambda^{2}} \\
d=d
\end{gathered}
$$

\section{Case 2:}

$$
\begin{gathered}
\alpha_{1}=0 \\
\alpha_{0}= \pm(\lambda-2 d(v-1)) \sqrt{\frac{q}{8 \mu(v-1)-2 \lambda^{2}}} \\
\alpha_{-1}= \pm 2\left(d^{2}(v-1)+\mu-\lambda d\right) \sqrt{\frac{q}{8 \mu(v-1)-2 \lambda^{2}}} \\
p=\frac{-2 q}{4 \mu(v-1)-\lambda^{2}} \\
d=d
\end{gathered}
$$

\section{Case 3:}

$$
\begin{gathered}
\alpha_{1}= \pm(v-1) \sqrt{\frac{q}{8 \mu(v-1)-2 \lambda^{2}}} \\
\alpha_{0}=0 \\
\alpha_{-1}=\mp \frac{\sqrt{q\left(4 \mu(v-1)-\lambda^{2}\right)}}{4 \sqrt{2}(v-1)} \\
p=\frac{-q}{8 \mu(v-1)-2 \lambda^{2}}
\end{gathered}
$$




$$
d=\frac{\lambda}{2(v-1)}
$$

\section{Case 4:}

$$
\begin{gathered}
\alpha_{1}= \pm(v-1) \sqrt{\frac{-2 q}{8 \mu(v-1)-2 \lambda^{2}}} \\
\alpha_{0}=0 \\
\alpha_{-1}=\mp \frac{\sqrt{-4 q \mu(v-1)-q \lambda^{2}}}{4(v-1)} \\
p=\frac{q}{4 \mu(v-1)-\lambda^{2}} \\
d=\frac{\lambda}{2(v-1)}
\end{gathered}
$$

where $p, q, d, \lambda, \mu$ and $v$ are arbitrary constants.

Substituting (3.7)-(3.10) into solution Eq.(3.5), we derive

$$
\begin{gathered}
u_{1}(z, t)= \pm(\lambda-2 d(v-1)) \sqrt{\frac{q}{8 \mu(v-1)-2 \lambda^{2}}} \\
\pm 2(v-1) \sqrt{\frac{q}{8 \mu(v-1)-2 \lambda^{2}}}\left(d+\left(G^{\prime} / G\right)\right)
\end{gathered}
$$

where $\xi=a z \mp \sqrt{\frac{4 \mu(v-1)-\lambda^{2}-2 q}{4 \mu(v-1)-\lambda^{2}}} t, p, q, d, \lambda, \mu$ and $v$ are arbitrary constants.

$$
\begin{gathered}
u_{2}(z, t)=\mp(\lambda-2 d(v-1)) \sqrt{\frac{q}{8 \mu(v-1)-2 \lambda^{2}}} \\
\pm 2\left(d^{2}(v-1)+\mu-\lambda d\right) \sqrt{\frac{q}{8 \mu(v-1)-2 \lambda^{2}}}\left(d+\left(G^{\prime} / G\right)\right)^{-1}
\end{gathered}
$$

where $\xi=a z \mp \sqrt{\frac{4 \mu(v-1)-\lambda^{2}-2 q}{4 \mu(v-1)-\lambda^{2}}} t, p, q, d, \lambda, \mu$ and $v$ are arbitrary constants.

$$
\begin{aligned}
u_{3}(z, t) & = \pm(v-1) \sqrt{\frac{q}{8 \mu(v-1)-2 \lambda^{2}}}\left(\frac{\lambda}{2(v-1)}+\left(G^{\prime} / G\right)\right) \\
\pm & \frac{\sqrt{q\left(4 \mu(v-1)-\lambda^{2}\right)}}{4 \sqrt{2}(v-1)}\left(\frac{\lambda}{2(v-1)}+\left(G^{\prime} / G\right)\right)^{-1}
\end{aligned}
$$

where $\xi=a z \mp \sqrt{\frac{8 \mu(v-1)-2 \lambda^{2}-q}{8 \mu(v-1)-2 \lambda^{2}}} t, p, q, d, \lambda, \mu$ and $v$ are arbitrary constants.

$$
\begin{gathered}
u_{4}(z, t)= \pm(v-1) \sqrt{\frac{-2 q}{8 \mu(v-1)-2 \lambda^{2}}}\left(\frac{\lambda}{2(v-1)}+\left(G^{\prime} / G\right)\right) \\
\mp \frac{\sqrt{-4 q \mu(v-1)-q \lambda^{2}}}{4(v-1)}\left(\frac{\lambda}{2(v-1)}+\left(G^{\prime} / G\right)\right)^{-1}
\end{gathered}
$$

where $\xi=a z \mp \sqrt{\frac{4 \mu(v-1)-\lambda^{2}+q}{4 \mu(v-1)-\lambda^{2}}} t, p, q, d, \lambda, \mu$ and $v$ are arbitrary constants. 
Substituting the value of $\left(\mathrm{G}^{\prime} / \mathrm{G}\right)$ into Eq.(3.14), we obtain solutions of Eq.(1.1) as follows: When $\Omega=\lambda^{2}-4 \mu v+4 \mu>0, \lambda(v-1) \neq 0$ (or $\left.\mu(v-1) \neq 0\right)$, we have

$$
\begin{aligned}
& u_{1_{1}}(z, t)= \pm(\lambda-2 d(v-1)) \sqrt{\frac{q}{8 \mu(v-1)-2 \lambda^{2}}} \pm 2(v-1) \sqrt{\frac{q}{8 \mu(v-1)-2 \lambda^{2}}} \\
& \times\left\{d-\frac{1}{2(v-1)}\left(\lambda+\sqrt{\Omega} \tanh \left(\frac{1}{2} \sqrt{\Omega} \xi\right)\right)\right\} \\
& u_{1_{2}}(z, t)= \pm(\lambda-2 d(v-1)) \sqrt{\frac{q}{8 \mu(v-1)-2 \lambda^{2}}} \pm 2(v-1) \sqrt{\frac{q}{8 \mu(v-1)-2 \lambda^{2}}} \\
& \times\left\{d-\frac{1}{2(v-1)}\left(\lambda+\sqrt{\Omega} \operatorname{coth}\left(\frac{1}{2} \sqrt{\Omega} \xi\right)\right)\right\} \\
& u_{1_{3}}(z, t)= \pm(\lambda-2 d(v-1)) \sqrt{\frac{q}{8 \mu(v-1)-2 \lambda^{2}}} \pm 2(v-1) \sqrt{\frac{q}{8 \mu(v-1)-2 \lambda^{2}}} \\
& \times\left\{d-\frac{1}{2(v-1)}(\lambda+\sqrt{\Omega}(\tanh (\sqrt{\Omega} \xi) \pm i \operatorname{sech}(\sqrt{\Omega} \xi)))\right\} \\
& u_{1_{4}}(z, t)= \pm(\lambda-2 d(v-1)) \sqrt{\frac{q}{8 \mu(v-1)-2 \lambda^{2}}} \pm 2(v-1) \sqrt{\frac{q}{8 \mu(v-1)-2 \lambda^{2}}} \\
& \times\left\{d-\frac{1}{2(v-1)}(\lambda+\sqrt{\Omega}(\operatorname{coth}(\sqrt{\Omega} \xi) \pm \operatorname{csch}(\sqrt{\Omega} \xi)))\right\} \\
& u_{1_{5}}(z, t)= \pm(\lambda-2 d(v-1)) \sqrt{\frac{q}{8 \mu(v-1)-2 \lambda^{2}}} \pm 2(v-1) \sqrt{\frac{q}{8 \mu(v-1)-2 \lambda^{2}}} \\
& \times\left\{d-\frac{1}{4(v-1)}\left(2 \lambda+\sqrt{\Omega}\left(\tanh \left(\frac{1}{4} \sqrt{\Omega} \xi\right)+\operatorname{coth}\left(\frac{1}{4} \sqrt{\Omega} \xi\right)\right)\right)\right\} \\
& u_{1_{6}}(z, t)= \pm(\lambda-2 d(v-1)) \sqrt{\frac{q}{8 \mu(v-1)-2 \lambda^{2}}} \pm 2(v-1) \sqrt{\frac{q}{8 \mu(v-1)-2 \lambda^{2}}} \\
& \times\left\{d+\frac{1}{2(v-1)}\left(-\lambda+\frac{ \pm \sqrt{\Omega\left(m_{1}^{2}+m_{2}^{2}\right)}-m_{1} \sqrt{\Omega} \cosh (\sqrt{\Omega} \xi)}{m_{1} \sinh (\sqrt{\Omega} \xi)+m_{2}}\right)\right\} \\
& u_{1_{7}}(z, t)= \pm(\lambda-2 d(v-1)) \sqrt{\frac{q}{8 \mu(v-1)-2 \lambda^{2}}} \pm 2(v-1) \sqrt{\frac{q}{8 \mu(v-1)-2 \lambda^{2}}} \\
& \times\left\{d+\frac{1}{2(v-1)}\left(-\lambda+\frac{ \pm \sqrt{\Omega\left(m_{1}^{2}+m_{2}^{2}\right)}+m_{1} \sqrt{\Omega} \cosh (\sqrt{\Omega} \xi)}{m_{1} \sinh (\sqrt{\Omega} \xi)+m_{2}}\right)\right\}
\end{aligned}
$$

where $m_{1}$ and $m_{2}$ are real non-zero constants.

$$
\begin{aligned}
u_{1_{8}}(z, t)= \pm(\lambda- & 2 d(v-1)) \sqrt{\frac{q}{8 \mu(v-1)-2 \lambda^{2}}} \pm 2(v-1) \sqrt{\frac{q}{8 \mu(v-1)-2 \lambda^{2}}} \\
& \times\left\{d+\frac{2 \mu \cosh \left(\frac{1}{2} \sqrt{\Omega} \xi\right)}{\sqrt{\Omega} \sinh \left(\frac{1}{2} \sqrt{\Omega} \xi\right)-\lambda \cosh \left(\frac{1}{2} \sqrt{\Omega} \xi\right)}\right\} \\
u_{1_{9}}(z, t)= \pm(\lambda- & 2 d(v-1)) \sqrt{\frac{q}{8 \mu(v-1)-2 \lambda^{2}}} \pm 2(v-1) \sqrt{\frac{q}{8 \mu(v-1)-2 \lambda^{2}}} \\
& \times\left\{d+\frac{2 \mu \sinh \left(\frac{1}{2} \sqrt{\Omega} \xi\right)}{\sqrt{\Omega} \cosh \left(\frac{1}{2} \sqrt{\Omega} \xi\right)-\lambda \sinh \left(\frac{1}{2} \sqrt{\Omega} \xi\right)}\right\}
\end{aligned}
$$




$$
\begin{aligned}
u_{1_{10}}(z, t)= \pm( & \lambda-2 d(v-1)) \sqrt{\frac{q}{8 \mu(v-1)-2 \lambda^{2}}} \pm 2(v-1) \sqrt{\frac{q}{8 \mu(v-1)-2 \lambda^{2}}} \\
& \times\left\{d+\frac{2 \mu \cosh (\sqrt{\Omega} \xi)}{\sqrt{\Omega} \sinh (\sqrt{\Omega} \xi)-\lambda \cosh (\sqrt{\Omega} \xi) \pm i \sqrt{\Omega}}\right\} \\
u_{1_{11}}(z, t)= \pm( & \lambda-2 d(v-1)) \sqrt{\frac{q}{8 \mu(v-1)-2 \lambda^{2}}} \pm 2(v-1) \sqrt{\frac{q}{8 \mu(v-1)-2 \lambda^{2}}} \\
& \times\left\{d+\frac{2 \mu \sinh (\sqrt{\Omega} \xi)}{\sqrt{\Omega} \cosh (\sqrt{\Omega} \xi)-\lambda \sinh (\sqrt{\Omega} \xi) \pm \sqrt{\Omega}}\right\}
\end{aligned}
$$

When $\Omega=\lambda^{2}-4 \mu v+4 \mu<0, \lambda(v-1) \neq 0$ (or $\left.\mu(v-1) \neq 0\right)$, we have

$$
\begin{aligned}
& u_{1_{12}}(z, t)= \pm(\lambda-2 d(v-1)) \sqrt{\frac{q}{8 \mu(v-1)-2 \lambda^{2}}} \pm 2(v-1) \sqrt{\frac{q}{8 \mu(v-1)-2 \lambda^{2}}} \\
& \times\left\{d+\frac{1}{2(v-1)}\left(-\lambda+\sqrt{-\Omega} \tan \left(\frac{1}{2} \sqrt{-\Omega} \xi\right)\right)\right\} \\
& u_{1_{13}}(z, t)= \pm(\lambda-2 d(v-1)) \sqrt{\frac{q}{8 \mu(v-1)-2 \lambda^{2}}} \pm 2(v-1) \sqrt{\frac{q}{8 \mu(v-1)-2 \lambda^{2}}} \\
& \times\left\{d-\frac{1}{2(v-1)}\left(\lambda+\sqrt{-\Omega} \cot \left(\frac{1}{2} \sqrt{-\Omega} \xi\right)\right)\right\} \\
& u_{1_{14}}(z, t)= \pm(\lambda-2 d(v-1)) \sqrt{\frac{q}{8 \mu(v-1)-2 \lambda^{2}}} \pm 2(v-1) \sqrt{\frac{q}{8 \mu(v-1)-2 \lambda^{2}}} \\
& \times\left\{d-\frac{1}{2(v-1)}(-\lambda+\sqrt{-\Omega}(\tan (\sqrt{-\Omega} \xi) \pm \sec (\sqrt{-\Omega} \xi)))\right\} \\
& u_{1_{15}}(z, t)= \pm(\lambda-2 d(v-1)) \sqrt{\frac{q}{8 \mu(v-1)-2 \lambda^{2}}} \pm 2(v-1) \sqrt{\frac{q}{8 \mu(v-1)-2 \lambda^{2}}} \\
& \times\left\{d-\frac{1}{2(v-1)}(\lambda+\sqrt{-\Omega}(\cot (\sqrt{-\Omega} \xi) \pm \csc (\sqrt{-\Omega} \xi)))\right\} \\
& u_{1_{16}}(z, t)= \pm(\lambda-2 d(v-1)) \sqrt{\frac{q}{8 \mu(v-1)-2 \lambda^{2}}} \pm 2(v-1) \sqrt{\frac{q}{8 \mu(v-1)-2 \lambda^{2}}} \\
& \times\left\{d+\frac{1}{4(v-1)}\left(-2 \lambda+\sqrt{-\Omega}\left(\tan \left(\frac{1}{4} \sqrt{-\Omega} \xi\right)-\cot \left(\frac{1}{4} \sqrt{-\Omega} \xi\right)\right)\right)\right\} \\
& u_{1_{17}}(z, t)= \pm(\lambda-2 d(v-1)) \sqrt{\frac{q}{8 \mu(v-1)-2 \lambda^{2}}} \pm 2(v-1) \sqrt{\frac{q}{8 \mu(v-1)-2 \lambda^{2}}} \\
& \times\left\{d+\frac{1}{2(v-1)}\left(-\lambda+\frac{ \pm \sqrt{-\Omega\left(m_{1}^{2}-m_{2}^{2}\right)}-m_{1} \sqrt{-\Omega} \cos (\sqrt{-\Omega} \xi)}{m_{1} \sin (\sqrt{-\Omega} \xi)+m_{2}}\right)\right\} \\
& u_{1_{18}}(z, t)= \pm(\lambda-2 d(v-1)) \sqrt{\frac{q}{8 \mu(v-1)-2 \lambda^{2}}} \pm 2(v-1) \sqrt{\frac{q}{8 \mu(v-1)-2 \lambda^{2}}} \\
& \times\left\{d+\frac{1}{2(v-1)}\left(-\lambda+\frac{ \pm \sqrt{-\Omega\left(m_{1}^{2}-m_{2}^{2}\right)}+m_{1} \sqrt{-\Omega} \cos (\sqrt{-\Omega} \xi)}{m_{1} \sin (\sqrt{-\Omega} \xi)+m_{2}}\right)\right\}
\end{aligned}
$$

where $m_{1}$ and $m_{2}$ are arbitrary constants such that $p^{2}-q^{2}>0$.

$$
u_{1_{19}}(z, t)= \pm(\lambda-2 d(v-1)) \sqrt{\frac{q}{8 \mu(v-1)-2 \lambda^{2}}} \pm 2(v-1) \sqrt{\frac{q}{8 \mu(v-1)-2 \lambda^{2}}}
$$




$$
\begin{aligned}
& \times\left\{d-\frac{2 \mu \cos \left(\frac{1}{2} \sqrt{-\Omega} \xi\right)}{\sqrt{-\Omega} \sin \left(\frac{1}{2} \sqrt{-\Omega} \xi\right)+\lambda \cos \left(\frac{1}{2} \sqrt{-\Omega} \xi\right)}\right\} \\
& u_{1_{20}}(z, t)= \pm(\lambda-2 d(v-1)) \sqrt{\frac{q}{8 \mu(v-1)-2 \lambda^{2}}} \pm 2(v-1) \sqrt{\frac{q}{8 \mu(v-1)-2 \lambda^{2}}} \\
& \times\left\{d+\frac{2 \mu \sin \left(\frac{1}{2} \sqrt{-\Omega} \xi\right)}{\sqrt{-\Omega} \cos \left(\frac{1}{2} \sqrt{-\Omega} \xi\right)-\lambda \sin \left(\frac{1}{2} \sqrt{-\Omega} \xi\right)}\right\} \\
& u_{1_{21}}(z, t)= \pm(\lambda-2 d(v-1)) \sqrt{\frac{q}{8 \mu(v-1)-2 \lambda^{2}}} \pm 2(v-1) \sqrt{\frac{q}{8 \mu(v-1)-2 \lambda^{2}}} \\
& \times\left\{d-\frac{2 \mu \cos (\sqrt{-\Omega} \xi)}{\sqrt{-\Omega} \sin (\sqrt{-\Omega} \xi)+\lambda \cos (\sqrt{-\Omega} \xi) \pm \sqrt{\Omega}}\right\} \\
& u_{1_{22}}(z, t)= \pm(\lambda-2 d(v-1)) \sqrt{\frac{q}{8 \mu(v-1)-2 \lambda^{2}}} \pm 2(v-1) \sqrt{\frac{q}{8 \mu(v-1)-2 \lambda^{2}}} \\
& \times\left\{d+\frac{2 \mu \sin \left(\frac{1}{2} \sqrt{-\Omega} \xi\right)}{\sqrt{-\Omega} \cos \left(\frac{1}{2} \sqrt{-\Omega} \xi\right)-\lambda \sin \left(\frac{1}{2} \sqrt{-\Omega} \xi\right) \pm \sqrt{\Omega}}\right\}
\end{aligned}
$$

When $\mu=0$ and $\lambda(v-1) \neq 0$,

$$
\begin{aligned}
u_{1_{23}}(z, t)= \pm(\lambda- & 2 d(v-1)) \sqrt{\frac{q}{8 \mu(v-1)-2 \lambda^{2}}} \pm 2(v-1) \sqrt{\frac{q}{8 \mu(v-1)-2 \lambda^{2}}} \\
& \times\left\{d-\frac{\lambda k}{(v-1)(k+\cosh (\lambda \xi)-\sinh (\lambda \xi))}\right\} \\
u_{1_{24}}(z, t)= \pm(\lambda- & 2 d(v-1)) \sqrt{\frac{q}{8 \mu(v-1)-2 \lambda^{2}}} \pm 2(v-1) \sqrt{\frac{q}{8 \mu(v-1)-2 \lambda^{2}}} \\
& \times\left\{d-\frac{\lambda(\cosh (\lambda \xi)+\sinh (\lambda \xi))}{(v-1)(k+\cosh (\lambda \xi)+\sinh (\lambda \xi))}\right\}
\end{aligned}
$$

where $k$ is an arbitrary constant.

Similarly, substituting the value of $\left(\mathrm{G}^{\prime} / \mathrm{G}\right)$ into Eq.(3.15), we achieve the solutions of Eq.(1.1):

When $\Omega=\lambda^{2}-4 \mu v+4 \mu>0, \lambda(v-1) \neq 0$ (or $\left.\mu(v-1) \neq 0\right)$, we get

$$
\begin{aligned}
& u_{2_{1}}(z, t)=\mp(\lambda-2 d(v-1)) \sqrt{\frac{q}{8 \mu(v-1)-2 \lambda^{2}}} \pm 2\left(d^{2}(v-1)+\mu-\lambda d\right) \sqrt{\frac{q}{8 \mu(v-1)-2 \lambda^{2}}} \\
& \times\left\{d-\frac{1}{2(v-1)}\left(\lambda+\sqrt{\Omega} \tanh \left(\frac{1}{2} \sqrt{\Omega} \xi\right)\right)\right\}^{-1} \\
& u_{2_{2}}(z, t)=\mp(\lambda-2 d(v-1)) \sqrt{\frac{q}{8 \mu(v-1)-2 \lambda^{2}}} \pm 2\left(d^{2}(v-1)+\mu-\lambda d\right) \sqrt{\frac{q}{8 \mu(v-1)-2 \lambda^{2}}} \\
& \times\left\{d-\frac{1}{2(v-1)}\left(\lambda+\sqrt{\Omega} \operatorname{coth}\left(\frac{1}{2} \sqrt{\Omega} \xi\right)\right)\right\}^{-1} \\
& u_{2_{3}}(z, t)=\mp(\lambda-2 d(v-1)) \sqrt{\frac{q}{8 \mu(v-1)-2 \lambda^{2}}} \pm 2\left(d^{2}(v-1)+\mu-\lambda d\right) \sqrt{\frac{q}{8 \mu(v-1)-2 \lambda^{2}}}
\end{aligned}
$$




$$
\times\left\{d-\frac{1}{2(v-1)}(\lambda+\sqrt{\Omega}(\tanh (\sqrt{\Omega} \xi) \pm i \operatorname{sech}(\sqrt{\Omega} \xi)))\right\}^{-1}
$$

The other families of exact solutions of Eq.(1.1) are omitted for convenience.

When $\Omega=\lambda^{2}-4 \mu v+4 \mu<0, \lambda(v-1) \neq 0($ or $\mu(v-1) \neq 0$ ), we get

$$
\begin{gathered}
u_{2_{12}}(z, t)=\mp(\lambda-2 d(v-1)) \sqrt{\frac{q}{8 \mu(v-1)-2 \lambda^{2}}} \pm 2\left(d^{2}(v-1)+\mu-\lambda d\right) \sqrt{\frac{q}{8 \mu(v-1)-2 \lambda^{2}}} \\
\left.\times\left\{d+\frac{1}{2(v-1)}\left(-\lambda+\sqrt{-\Omega} \tan \left(\frac{1}{2} \sqrt{-\Omega} \xi\right)\right)\right)\right\}^{-1} \\
u_{2_{13}}(z, t)=\mp(\lambda-2 d(v-1)) \sqrt{\frac{q}{8 \mu(v-1)-2 \lambda^{2}}} \pm 2\left(d^{2}(v-1)+\mu-\lambda d\right) \sqrt{\frac{q}{8 \mu(v-1)-2 \lambda^{2}}} \\
\left.\times\left\{d-\frac{1}{2(v-1)}\left(\lambda+\sqrt{-\Omega} \cot \left(\frac{1}{2} \sqrt{-\Omega} \xi\right)\right)\right)\right\}^{-1}
\end{gathered}
$$

The other families of exact solutions of Eq.(1.1) are omitted for convenience.

When $\mu=0$ and $\lambda(v-1) \neq 0$,

$$
\begin{gathered}
u_{2_{23}}(z, t)=\mp(\lambda-2 d(v-1)) \sqrt{\frac{q}{8 \mu(v-1)-2 \lambda^{2}}} \pm 2\left(d^{2}(v-1)+\mu-\lambda d\right) \sqrt{\frac{q}{8 \mu(v-1)-2 \lambda^{2}}} \\
\times\left\{d-\frac{\lambda k}{(v-1)(k+\cosh (\lambda \xi)-\sinh (\lambda \xi))}\right\}^{-1}
\end{gathered}
$$

where $k$ is an arbitrary constant.

The other families of exact solutions of Eq.(1.1) are omitted for convenience.

Again, substituting the value of (G'/G) into Eq.(3.16), we achieve the solutions Eq.(1.1):

When $\Omega=\lambda^{2}-4 \mu v+4 \mu>0, \lambda(v-1) \neq 0($ or $\mu(v-1) \neq 0)$, we receive

$$
\begin{gathered}
u_{3_{1}}(z, t)= \pm(v-1) \sqrt{\frac{q}{8 \mu(v-1)-2 \lambda^{2}}} \times\left\{\frac{\lambda}{2(v-1)}-\frac{1}{2(v-1)}\left(\lambda+\sqrt{\Omega} \tanh \left(\frac{1}{2} \sqrt{\Omega} \xi\right)\right)\right\} \\
\pm \frac{\sqrt{q\left(4 \mu(v-1)-\lambda^{2}\right)}}{4 \sqrt{2}(v-1)} \times\left\{\frac{\lambda}{2(v-1)}-\frac{1}{2(v-1)}\left(\lambda+\sqrt{\Omega} \tanh \left(\frac{1}{2} \sqrt{\Omega} \xi\right)\right)\right\}^{-1} \\
u_{3_{2}}(z, t)= \pm(v-1) \sqrt{\frac{q}{8 \mu(v-1)-2 \lambda^{2}}} \times\left\{\frac{\lambda}{2(v-1)}-\frac{1}{2(v-1)}\left(\lambda+\sqrt{\Omega} \operatorname{coth}\left(\frac{1}{2} \sqrt{\Omega} \xi\right)\right)\right\} \\
\pm \frac{\sqrt{q\left(4 \mu(v-1)-\lambda^{2}\right)}}{4 \sqrt{2}(v-1)} \times\left\{\frac{\lambda}{2(v-1)}-\frac{1}{2(v-1)}\left(\lambda+\sqrt{\Omega} \operatorname{coth}\left(\frac{1}{2} \sqrt{\Omega} \xi\right)\right)\right\}^{-1}
\end{gathered}
$$

The other families of exact solutions of Eq.(1.1) are omitted for convenience.

When $\Omega=\lambda^{2}-4 \mu v+4 \mu<0, \lambda(v-1) \neq 0$ (or $\mu(v-1) \neq 0$ ), we receive

$$
\begin{gathered}
u_{3_{12}}(z, t)= \pm(v-1) \sqrt{\frac{q}{8 \mu(v-1)-2 \lambda^{2}}} \times\left\{\frac{\lambda}{2(v-1)}+\frac{1}{2(v-1)}\left(-\lambda+\sqrt{-\Omega} \tan \left(\frac{1}{2} \sqrt{-\Omega} \xi\right)\right)\right\} \\
\pm \frac{\sqrt{q\left(4 \mu(v-1)-\lambda^{2}\right)}}{4 \sqrt{2}(v-1)} \times\left\{\frac{\lambda}{2(v-1)}+\frac{1}{2(v-1)}\left(-\lambda+\sqrt{-\Omega} \tan \left(\frac{1}{2} \sqrt{-\Omega} \xi\right)\right)\right\}^{-1} \\
u_{3_{13}}(z, t)= \pm(v-1) \sqrt{\frac{q}{8 \mu(v-1)-2 \lambda^{2}}} \times\left\{\frac{\lambda}{2(v-1)}-\frac{1}{2(v-1)}\left(\lambda+\sqrt{-\Omega} \cot \left(\frac{1}{2} \sqrt{-\Omega} \xi\right)\right)\right\}
\end{gathered}
$$




$$
\pm \frac{\sqrt{q\left(4 \mu(v-1)-\lambda^{2}\right)}}{4 \sqrt{2}(v-1)} \times\left\{\frac{\lambda}{2(v-1)}-\frac{1}{2(v-1)}\left(\lambda+\sqrt{-\Omega} \cot \left(\frac{1}{2} \sqrt{-\Omega} \xi\right)\right)\right\}^{-1}
$$

The other families of exact solutions of Eq.(1.1) are omitted for convenience.

When $\mu=0, \lambda(v-1) \neq 0$, we receive

$$
\begin{gathered}
u_{3_{23}(z, t)}= \pm(v-1) \sqrt{\frac{q}{8 \mu(v-1)-2 \lambda^{2}}} \times\left\{\frac{\lambda}{2(v-1)}-\frac{\lambda k}{(v-1)(k+\cosh (\lambda \xi)-\sinh (\lambda \xi))}\right\} \\
\pm \frac{\sqrt{q\left(4 \mu(v-1)-\lambda^{2}\right)}}{4 \sqrt{2}(v-1)} \times\left\{\frac{\lambda}{2(v-1)}-\frac{\lambda k}{(v-1)(k+\cosh (\lambda \xi)-\sinh (\lambda \xi))}\right\}^{-1}
\end{gathered}
$$

where $k$ is an arbitrary constant.

The other families of exact solutions of Eq.(1.1) are omitted for convenience.

Finally, substituting the value of $\left(\mathrm{G}^{\prime} / \mathrm{G}\right)$ into Eq.(3.17), we achieve the solutions Eq.(1.1):

When $\Omega=\lambda^{2}-4 \mu v+4 \mu>0, \lambda(v-1) \neq 0($ or $\mu(v-1) \neq 0)$, we know

$$
\begin{gathered}
u_{4_{1}}(z, t)= \pm(v-1) \sqrt{\frac{-2 q}{8 \mu(v-1)-2 \lambda^{2}} \times\left\{\frac{\lambda}{2(v-1)}-\frac{1}{2(v-1)}\left(\lambda+\sqrt{\Omega} \tanh \left(\frac{1}{2} \sqrt{\Omega} \xi\right)\right)\right\}} \\
\mp \frac{\sqrt{-4 q \mu(v-1)-q \lambda^{2}}}{4(v-1)} \times\left\{\frac{\lambda}{2(v-1)}-\frac{1}{2(v-1)}\left(\lambda+\sqrt{\Omega} \tanh \left(\frac{1}{2} \sqrt{\Omega} \xi\right)\right)\right\}^{-1} \\
u_{4_{2}}(z, t)= \pm(v-1) \sqrt{\frac{-2 q}{8 \mu(v-1)-2 \lambda^{2}}} \times\left\{\frac{\lambda}{2(v-1)}-\frac{1}{2(v-1)}\left(\lambda+\sqrt{\Omega} \operatorname{coth}\left(\frac{1}{2} \sqrt{\Omega} \xi\right)\right)\right\} \\
\mp \frac{\sqrt{-4 q \mu(v-1)-q \lambda^{2}}}{4(v-1)} \times\left\{\frac{\lambda}{2(v-1)}-\frac{1}{2(v-1)}\left(\lambda+\sqrt{\Omega} \operatorname{coth}\left(\frac{1}{2} \sqrt{\Omega} \xi\right)\right)\right\}^{-1}
\end{gathered}
$$

The other families of exact solutions of Eq.(1.1) are omitted for convenience.

When $\Omega=\lambda^{2}-4 \mu v+4 \mu<0, \lambda(v-1) \neq 0($ or $\mu(v-1) \neq 0)$, we know

$$
\begin{gathered}
u_{4_{12}}(z, t)= \pm(v-1) \sqrt{\frac{-2 q}{8 \mu(v-1)-2 \lambda^{2}}} \times\left\{\frac{\lambda}{2(v-1)}+\frac{1}{2(v-1)}\left(-\lambda+\sqrt{-\Omega} \tan \left(\frac{1}{2} \sqrt{-\Omega} \xi\right)\right)\right\} \\
\mp \frac{\sqrt{-4 q \mu(v-1)-q \lambda^{2}}}{4(v-1)} \times\left\{\frac{\lambda}{2(v-1)}+\frac{1}{2(v-1)}\left(-\lambda+\sqrt{-\Omega} \tan \left(\frac{1}{2} \sqrt{-\Omega} \xi\right)\right)\right\}^{-1} \\
u_{4_{13}}(z, t)= \pm(v-1) \sqrt{\frac{-2 q}{8 \mu(v-1)-2 \lambda^{2}}} \times\left\{\frac{\lambda}{2(v-1)}-\frac{1}{2(v-1)}\left(\lambda+\sqrt{-\Omega} \cot \left(\frac{1}{2} \sqrt{-\Omega} \xi\right)\right)\right\} \\
\mp \frac{\sqrt{-4 q \mu(v-1)-q \lambda^{2}}}{4(v-1)} \times\left\{\frac{\lambda}{2(v-1)}-\frac{1}{2(v-1)}\left(\lambda+\sqrt{-\Omega} \cot \left(\frac{1}{2} \sqrt{-\Omega} \xi\right)\right)\right\}^{-1}
\end{gathered}
$$

The other families of exact solutions of Eq.(1.1) are omitted for convenience.

When $\mu=0$ and $\lambda(v-1) \neq 0$, we know

$$
\begin{gathered}
u_{4_{23}(z, t)}= \pm(v-1) \sqrt{\frac{-2 q}{8 \mu(v-1)-2 \lambda^{2}}} \times\left\{\frac{\lambda}{2(v-1)}-\frac{\lambda k}{(v-1)(k+\cosh (\lambda \xi)-\sinh (\lambda \xi))}\right\} \\
\mp \frac{\sqrt{-4 q \mu(v-1)-q \lambda^{2}}}{4(v-1)} \times\left\{\frac{\lambda}{2(v-1)}-\frac{\lambda k}{(v-1)(k+\cosh (\lambda \xi)-\sinh (\lambda \xi))}\right\}^{-1}
\end{gathered}
$$

where $k$ is an arbitrary constant.

The other families of exact solutions of Eq.(1.1) are omitted for convenience. 


\section{Conclusion}

In this paper, we have used the novel $\left(\frac{G^{\prime}}{G}\right)$-expansion method to obtain some exact traveling wave solutions for generalized Davey-Stewartson equations with arbitrary power nonlinearities. Comparing the results with the effective result of [35]-[41] by Maple, we know that our results are new. Hence, the performance of this method is reliable. Finally, it also can be applied to solve other nonlinear PDEs for deriving some new exact solutions.

\section{References}

1. Baojian, Hong, Dianchen, Lu, Fushu and Sun, "The extended Jacobi Elliptic Functions expansion method and new exact solutions for the Zakharov equations," World Journal of Modelling and Simulation, 2009, 5(3). 216-224.

2. F.Loete, Q.Zhang and M.Sorine, "Experimental Evaluation of the Inverse Scattering Method for Electrical Cable Fault Diagnosis," IFAC-PapersOnLine, 2015,48. 766-771.

3. S A El-Wakil, A R Degheidy, E M Abulwafa, M A Madkour, M T Attia and M A Abdou, "Exact traveling wave solutions of Generalized Zakharov Equations with Arbitrary Power Nonlinearities," Imternational Journal of Nonlinear Science, 2009, 7(4). 455-461.

4. Xu-dong Yang and Hang-yu Ruan, "HBFGen: A maple package to construct the Hirota bilinear form for nonlinear equations," Applied Mathematics and Computation, 2013, 219(15). 8018-8025.

5. A. J. M. Jawad, M. Mirzazadeh, Q. Zhou and A. Biswas, "Optical solitons with anti-cubic nonlinearity using three integration schemes," superlattices and microstructures, 2017, 105. 1-10.

6. M. Mirzazadeh, M. Ekici, Q. Zhou and A. Biswas, "Exact solitons to generalized resonant dispersive nonlinear schrödinger equation with power law nonlinearity," optik, 2017, 130. 178-183.

7. E. M. E. Zayed and Abdul-Ghani Al-Nowehy, "The modified simple equation method, the exp-function method and the method of soliton ansatz for solving the long-short wave resonance equations," Z. Naturforsch, 2016, 71a. 103-112.

8. A. H. Arnous, M. Z. Ullah, M. Asma, S. P. Moshokoa, Q. Zhou, M. Mirzazadeh, A. Biswas and M. Belic, "Dark and singular dispersive optical solitons of schrödinger-Hirota equation by modified simple equation method," Optik, 2017, 136. 445-450.

9. M. Ekici, M. Mirzazadeh, A. Sonmezoglu, Q. Zhou, H. Triki, M. Z. Ullah, S. P. Moshokoa and A. Biswas, "Optical solitons in birefringent fibers with Kerr nonlinearity by exp-function method," Optik, 2017, 131. 964-976.

10. E. M. E. Zayed and Abdul-Ghani Al-Nowehy, "Exact solutions for nonlinear foam drainage equation," Indian J. Phys, 2017,91. 209-218.

11. E. M. E. Zayed and Abdul-Ghani Al-Nowehy, "Solitons and other solutions for the generalized KdV-mKdV equation with higher-order nonlinear terms," J. Partial Differ, Equ. 2016, 29. 218-245.

12. M. M. El-Borai, H. M. El-Owaidy, H. M. Ahmed, A. H. Arnous, S. Moshokoa and A. Biswas, M. Belic, "Dark and singular optical solitons with spatio-temporal dispersion using modified simple equation method," optik, 2017, 130. 324-331.

13. E. M. E. Zayed and Abdul-Ghani Al-Nowehy, "Exact solutions of the Biswas-Milovic equation, the ZK(m, $\mathrm{n}, \mathrm{k})$ equation and the $\mathrm{K}(\mathrm{m}, \mathrm{n})$ equation using the generalized Kudryashov method," open phys, $2016,14$. 129-139.

14. M. M. El-Borai, H. M. El-Owaidy, H. M. Ahmed, A. H. Arnous, S. P. Moshokoa, A.Biswas and M. Belic, "Topological and singular soliton to Kundu-Eckhaus equation with extended Kudryashov's method," optik, 2017, 128. 57-62.

15. M. Mirzazadeh, M. Ekici, A. Sonomezoglu, M. Eslami, Q. Zhou, A. H. Kara, D. Milovic, F. B. Majid, A. Biswas and M. Belic, "Optical solitons with complex Ginzburg-Landau equation," Nonlinear Dyna, $2016,85$. 1979-2016.

16. Q. Zhou, X. Xiong, Q. Zhu, Y. Liu, H. Yu, P. Yao, A. Biswas and M. Belicd, "Optical solitons with nonlinear dispersion in polynomial law medium, J. Optoelectron," Adv. Mater, 2015, 17. 82-86.

17. M. Savescu, A. H. Kara, S. Kumar, E. V. Krishnan, M. Z. Ullah, S. P. Moshokoa, Q. Zhou and A. Biswas, "Embedded solitons and conservation law with $\chi^{2}$ and $\chi 3$ nonlinear susceptibilities," Acta Physica Polonica A, 2017, 131. 297-303.

18. J. V. Guzman, M. Z. Ullah, M. Asma, Q. Zhou and A. Biswas, "Dispersive solitons in magneto optic waveguides," Superlattices and microstructures, 2017, 103. 161-170.

19. A. Biswas, M. Z. Ullah, M. Asma, Q. Zhou, S. P. Moshokoa and M. Belic, "Optical solitons with quadratic-cubic nonlinearity by semi-inverse variational principle," Optik, 2017, 139. 16-19. 
20. M. Z. Ullah, A. Biswas, S. P. Moshokoa, Q. Zhou, M. Mirzazadeh and M. Belic, "Dispersive optical solitons in DWDM systems," Optik, 2017, 132. 210-215.

21. H. O. Bakodah, A. A. Al Qarni, M. A. Banaja, Q. Zhou, S. P. Moshokoa and A. Biswas, "Bright and dark thirring optical solitons with improved adomian decomposition scheme," Optik, 2017, 130. 1115-1123.

22. M. A. Banaja, A. A. Al Qarni, H. O. Bakodah, Q. Zhou, S. P. Moshokoa and A. Biswas, "The investigate of optical solitons in cascaded system by improved adomian decomposition scheme," Optik, 2017, 130. 1107-1114.

23. H. Triki, F. Azzouzi, A. Biswas, S. P. Moshokoa and M. Belic, "Bright optical solitons with Kerr law nonlinearity and fifth order dispersion," Optik, 2017, 128. 172-177.

24. H. Triki, A. Biswas, S. P. Moshokoa and M. Belic, "Dipole solitons in optical metamaterials with Kerr law nonlinearity," Optik, 2017, 128. 71-76.

25. H. Triki, A. Biswas, S. P. Moshokoa and M. Belic, "Optical solitons and conservation laws with quadratic-cubic nonlinearity," Optik, 2017, 128. 63-70.

26. J. V. Guzman, M. F. Mahmood, Q. Zhou, H. Triki, A. H. Arnous, A. Biswas, S. P. Moshokoa and M. Belic, "Solitons in nonlinear directional couplers with optical metamaterials," Nonlinear Dyna, 2017, 87. 427-458.

27. H. Triki, A. H. Kara, A. Biswas, S. P. Moshokoa and M. Belic, "Optical solitons and conservation laws with anti-cubic nonlinearity," Optik, 2016, 127. 12056-12062.

28. A. H. Kara, A. Biswas and M. Belic, "Conservation laws for optical solitons in birefringent fibers and magneto-optic waveguides," Optik, 2016, 127. 11662-11673.

29. E. M. E. Zayed and Abdul-Ghani Al-Nowehy, "Exact solutions and optical soliton solutions for the $(2+1)-$ dimensional hyperbolic nonlinear Schrödinger equation," Optik, 2016, 127. 4970-4983.

30. M. Ekici, A. Sonmezoglu, Q. Zhou, S. P. Moshokoa, M. Z. Ullah, A. Biswas and M. Belic, "Optical solitons with DWDM technology and four-wave mixing by extended trial equation method," Superlattices and Microstructures, 2017, 107. 254-266.

31. M. Ekici, A. Sonmezoglu, Q. Zhou, S. P. Moshokoa, M. Z. Ullah, A. Biswas and M. Belic, "Solitons in magneto-optic waveguides by extended trial function scheme," Superlattices and Microstruc tures, 2017, 107. 197-218.

32. M. Ekici, M. Mirzazadeh, A. Sonmezoglu, M. Z. Ullah, Q. Zhou, H. Triki, S. P. Moshokoa and A. Biswas, "Optical solitons with anti-cubic nonlinearity by extended trial equation method," Optik, 2017, 136. 368-373.

33. Kudryashov NA, "A note on the $\left(G^{\prime} / G\right)$-expansion method," Appl Math Comput, 2010, 217. 1755-1768.

34. M.G.Hafez, Md.Nur Alam and M.Ali Akbar, "Exact traveling wave solutions to the Klein-Gordon equation using the novel (G'/G)-expansion method," Results in Physics, 2014, 4. 177-184.

35. Song M and Liu Z R, "Qualitative analysis and explicit traveling wave solutions for the Davey-Stewartson equation," Math methods appl sci, 2014, 37. 393-401.

36. Cao J and Lu H Y, "Exact traveling wave solutions of the generalized Davey-Stewartson equation," Journal of Shanghai normal university(natural sciences), 2015, 44. 330-338.

37. M Mirzazadeh, "Soliton solutions of Davey-Stewartson equation by trial equation method and ansatz approach," Nonlinear dynamics, 2015, 1-6.

38. Ming Song and Anjan Biswas, "Topological defects and bifurcation analysis of the DS Equation with power law nonlinearity," Applied mathematics and information sciences, 2015, 1719-1724.

39. Reza Farshbaf Zinati and Jalil Manafian, "Applications of He's semi-inverse method, ITEM and GGM to the Davey-Stewartson equation," The european physical journal plus, 2017, 132. 155.

40. Seyma Tuluce Demiray and Hasan Bulut, "New soliton solutions of Davey-Stewartson equation with power-law nonlinearity," Optical and quantum electronics march, 2017, 49. 117.

41. Mehdi Fazli Aghdaei and Hojatollah Adibi, "New methods to solve the resonant nonlinear schrödinger equation with time-dependent coefficients," Optical and quantum electronics, 2017, 49. 316.

42. Zhu S, "The generalized Riccati equation mapping method in nonlinear evolution equation, Application to (2+1)-dimensional boiti-leon-pempinelle equation," Chaos soliton fract, 2008, 37. 1335-422. 